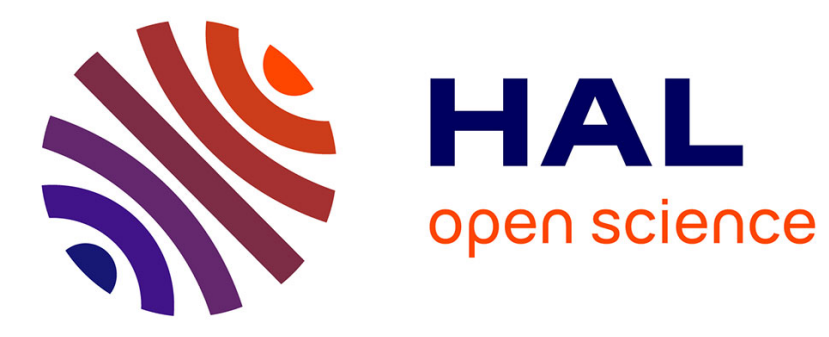

\title{
3-DOF potential air flow manipulation by inverse modeling control.
}

Anne Delettre, Guillaume J. Laurent, Nadine Le Fort-Piat, Christophe Varnier

\section{To cite this version:}

Anne Delettre, Guillaume J. Laurent, Nadine Le Fort-Piat, Christophe Varnier. 3-DOF potential air flow manipulation by inverse modeling control.. IEEE International Conference on Automation Science and Engineering, CASE'12., Aug 2012, Séoul, South Korea. pp.926-931. hal-00734018

\section{HAL Id: hal-00734018 https://hal.science/hal-00734018}

Submitted on 20 Sep 2012

HAL is a multi-disciplinary open access archive for the deposit and dissemination of scientific research documents, whether they are published or not. The documents may come from teaching and research institutions in France or abroad, or from public or private research centers.
L'archive ouverte pluridisciplinaire HAL, est destinée au dépôt et à la diffusion de documents scientifiques de niveau recherche, publiés ou non, émanant des établissements d'enseignement et de recherche français ou étrangers, des laboratoires publics ou privés. 


\title{
3-DOF potential air flow manipulation by inverse modeling control
}

\author{
Anne Delettre, Guillaume J. Laurent, Nadine Le Fort-Piat and Christophe Varnier
}

\begin{abstract}
Potential air flows can be used to perform nonprehensile contactless manipulations of objects gliding on airhockey table. In this paper, we introduce a general method able to perform 3-DOF position control of an object with potential air flow manipulators. This approach is based on an inverse modeling control scheme to perform closed-loop position servoing. We propose to use a linear programming algorithm to determine which sinks have to be activated in order to produce the suitable potential air flow to obtain the desired object motion. This approach is then validated on an experimental manipulator.
\end{abstract}

\section{INTRODUCTION}

Researchers have experimented a variety of air-jet techniques to design non-prehensile contactless manipulators. Most of them use are based on air bearing levitation. The sample is held on a plate which is drilled by many small holes. Pressurized air flows upward through these holes and creates an air cushion that counterbalances the weight of the component. This is the principle of popular air-hockey tables. Then, two approaches can be distinguished to move the object: tilted air jets and potential air flow.

Many devices use arrays of tilted air jets to produce a thrust force in addition to the air cushion. Some devices are designed to get stable transport system without closedloop control [1]. In contrast, the Xerox PARC paper handling system [2] uses 1,152 directed air jets in a 12 in. $\times 12$ in. array to levitate paper sheets. Each jet is separately controlled by an independent MEMS-like valve. Rij et al. [3] proposed a similar wafer transport system based on viscous traction principle. On a near microscopic scale, some active surfaces have been developed using MEMS actuators arrays. The surface of Fukuta et al. [4] is able to produce tilted air jets thanks to integrated electrostatic valves. Recently, Zeggari et al. [5] presented a passive pneumatic micro-conveyor that generates arrays of titled air jets for fast transport.

Luntz and Moon [6] introduced the use of potential air flow to move an object on an air-hockey table. They used a few flow sinks (suction points) above the table to create a stable flow pattern. More recently, they proposed methods to predict stable equilibria of an object freely moving on the table [7], [8], [9]. In previous works, we proposed to use vertical air jets rather than suction nozzles to induce potential air flow on an air-hockey table [10], [11]. A vertical air jet creates a local suction effect at its basement similarly to an

This work was supported by the Smart Blocks NRA (French National Research Agency) project (ANR-2011-BS03-005)

A. Delettre, G. J. Laurent, N. Le Fort-Piat and C. Varnier are with the Automatic Control and Micro-Mechatronic Systems Department, FEMTOST Institute, UFC-ENSMM-UTBM-CNRS, Université de Franche-Comté, Besançon, France, $\{$ Firstname. Lastname $\}$ dens $2 m$. fr air sink. We also proposed a method to control the position of an object along two degrees-of-freedom (2-DOF) of the plan. This method uses a superposition of patterns to induce a potential air flow in the required direction [12].

In this paper, we introduce a general method able to perform 3-DOF position control of an object with potential air flow manipulators. We propose to use a linear programming algorithm to determine which suction points have to be activated in order to produce the suitable potential air flow to obtain the desired motion of the object. This method is used in an inverse modeling control scheme to perform closed-loop position servoing.

Section II introduces the analytic model of the velocity field of the potential air flow according to the spatial configuration of vertical air jets. Then, Section III presents the inverse modeling control using linear programming. The obtained solutions are analyzed in Section IV. Finally, the method is validated on an experimental manipulator in Section V.

\section{Potential Air Flow MAnipulation}

In order to appreciate the ability of air-jet arrays to create potential air flow, it is first necessary to understand the basic characteristics of a single air-jet.

\section{A. Air-jet fundamentals}

The fundamental characteristics of turbulent gas jets have been described by Abramovich [13]. In the simplest case of a jet discharging fluid with a uniform initial velocity field $U_{e}$ into a motionless medium, the boundary layer thickness in the initial section (with diameter $D$ ) of the jet is zero. The boundary layer thickens away from the discharge point as particles of the surrounding medium become entrained and are carried along with corresponding particles of the jet which are slowed down. Whilst this leads to an increase in cross-section of the jet it also gradually "consumes" the nonviscous core. This short region of the jet in which the center line velocity remains constant is called the zone of flow establishment. Beyond this point, in the zone of established flow, the center line velocity of the jet $U_{m}$ gradually reduces as the radius $b(z)$ of the jet continues to expand linearly $(b(z)=0.114 z)$ :

$$
U_{m}=U_{e} \frac{D}{\sqrt{2} b(z)}
$$

In this area, the axial velocity profile is then:

$$
U(z, r)=U_{m} \exp \left[-\frac{r^{2}}{b^{2}(z)}\right]
$$


where $(z, r)$ are the axial and radial coordinates.

The volume flux is:

$$
\begin{aligned}
Q(z, r) & =\int_{0}^{r} 2 \pi y U(z, y) d y \\
& =\frac{\pi U_{e} D}{\sqrt{2}}\left(1-\exp \left[-\frac{r^{2}}{b^{2}(z)}\right]\right) b(z)
\end{aligned}
$$

The inflowing entrainment flow at $r$ (i.e. the suction strength) is then:

$$
\Lambda(r)=\frac{d}{d z} Q(z, r)
$$

For $r>b, \Lambda$ is nearly constant:

$$
\Lambda_{r>b} \approx \frac{0.114 \pi U_{e} D}{\sqrt{2}}
$$

The entrainment velocity for $r>b$ is then:

$$
U_{i}(r)=-\frac{\Lambda}{2 \pi r} \approx-\frac{0.114 U_{e} D}{2 \sqrt{2} r}
$$

The conclusion is that a vertical air jet can be assimilated to a sink (suction point) when $r>b$.

\section{B. Potential flow fields}

Assuming that the fluid is inviscid (that can be considered as true a few millimeters away from the orifice) and incompressible, the potential flow theory [14] predicts flow patterns depending on the position of the suction points (here the air jets).

Indeed, the velocity vector field $\vec{U}_{i}$ is equal to the negative gradient of the two dimensional scalar potential function $\Phi$ :

$$
\vec{U}_{i}=-\vec{\nabla} \Phi
$$

According to Eq. (7), the potential function $\Phi$ is given by:

$$
\Phi=\sum_{i=1}^{k} \frac{\Lambda_{i}}{2 \pi} \ln \left(r_{i}\right)
$$

where $\Lambda_{i}$ is the strength of the $i^{\text {th }}$ sink given by Eq. (6) and $r_{i}$ is the distance from the $i^{t h}$ sink. The velocity vector flow fields can then be re-written as a sum:

$$
\vec{U}_{i}=-\sum_{i=1}^{k} \frac{\Lambda_{i}}{2 \pi r_{i}} \vec{e}_{r, i}
$$

where $\vec{e}_{r, i}$ is the unit vector which gives the direction from the $i^{t h}$ sink to the object center.

\section{Aerodynamic forces modeling}

1) Force: The main force experienced by the object in a laminar flow is the skin friction, given by:

$$
\vec{F}=\iint b \overrightarrow{V_{r e l, P}} d S=\overrightarrow{F_{a i r}}+\overrightarrow{F_{P}}
$$

where $b$ is the skin friction coefficient, $V_{r e l, P}$ is the velocity of the fluid relative to the velocity of a point $P$ belonging to the object, $S$ is the surface of the horizontal face of the object, $U_{i, P}$ is the velocity of the air at point $P$ and $V_{P}$ is the velocity of point $P$. The skin friction coefficient $b$ depends

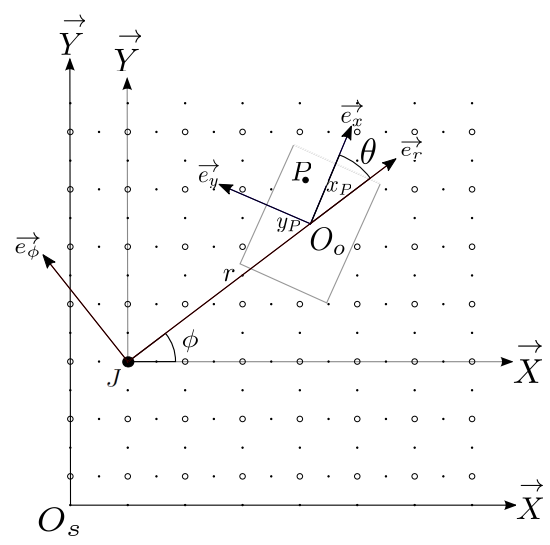

Fig. 1. Notations.

on the physical and geometrical properties of the object. Both terms in Eq. (11) can be calculated separately. These integrals depend on the horizontal surface $S$ of the object. We have calculated them analytically in the case of a rectangular shape, but we can do it (analytically or not) for any shape.

The force $F_{P}$ linked to the point $P$ velocity is given by :

$$
\overrightarrow{F_{P}}=\iint-b \overrightarrow{V_{P}} d S=-b L l \overrightarrow{V_{O_{o}}}
$$

where $V_{O_{o}}$ is velocity of the center $O_{o}$ of the object, and $L$ and $l$ are the dimensions of the surface $S$ of the object.

For only one active sink (represented by $J$ on Fig. 1, the force due to the air can be deduced from the air velocity $U_{i, P}$ (Eq. (10)):

$$
\overrightarrow{F_{a i r}}=\iint b \overrightarrow{U_{i, P}} d S=-\int_{\frac{-l}{2}}^{\frac{l}{2}} \int_{\frac{-L}{2}}^{\frac{L}{2}} \frac{b \Lambda_{i}}{2 \pi d_{i}} \overrightarrow{e_{d, i}} d x_{P} d y_{P}
$$

where $d_{i}$ is the distance between the point $P$ and the origin $J$ of the sink, $\overrightarrow{e_{d, i}}$ is the direction from $J$ to $P$, and $\left(x_{P}, y_{P}\right)$ are the coordinates of $P$ in the coordinate frame $\left(O_{o}, \overrightarrow{e_{x}}, \overrightarrow{e_{y}}\right)$ linked to the principal axis of the object (Fig. 1).

The next step is to express the force in the polar coordinate frame $\left(J, \overrightarrow{e_{r}}, \overrightarrow{e_{\phi}}\right)$, defined such as $\overrightarrow{e_{r}} \cdot \overrightarrow{e_{x}}=\cos \theta$, where $\theta$ is the orientation of the object in this frame. By the change of variables:

$$
\left\{\begin{array} { l } 
{ u = x _ { P } + r \operatorname { c o s } \theta } \\
{ v = y _ { P } - r \operatorname { s i n } \theta }
\end{array} \Leftrightarrow \left\{\begin{array}{l}
u_{1,2}= \pm \frac{L}{2}+r \cos \theta \\
v_{1,2}= \pm \frac{l}{2}-r \sin \theta
\end{array}\right.\right.
$$

Eq. (13) becomes:

$$
\vec{F}_{\text {air } /\left(J, \overrightarrow{e_{r}}, \overrightarrow{e_{\phi}}\right)}=\left[\begin{array}{l}
-\frac{b \Lambda}{2 \pi}\left(f_{1} \cos \theta-f_{2} \sin \theta\right) \\
-\frac{b \Lambda}{2 \pi}\left(f_{1} \sin \theta-f_{2} \cos \theta\right)
\end{array}\right]
$$

where:

$$
\begin{aligned}
f_{1} & =\frac{1}{2} v_{2} \ln \left(u_{2}^{2}+v_{2}^{2}\right)+u_{2} \tan ^{-1} \frac{v_{2}}{u_{2}}-\frac{1}{2} v_{2} \ln \left(u_{1}^{2}+v_{2}^{2}\right) \ldots \\
& -u_{1} \tan ^{-1} \frac{v_{2}}{u_{1}}-\frac{1}{2} v_{1} \ln \left(u_{2}^{2}+v_{1}^{2}\right)-u_{2} \tan ^{-1} \frac{v_{1}}{u_{2}} \ldots \\
& +\frac{1}{2} v_{1} \ln \left(u_{1}^{2}+v_{1}^{2}\right)+u_{1} \tan ^{-1} \frac{v_{1}}{u_{1}}
\end{aligned}
$$


and $f_{2}\left(u_{1}, u_{2}, v_{1}, v_{2}\right)$ is with a similarly form.

The last step is to express the force in the global coordinate frame $\left(O_{s}, \vec{X}, \vec{Y}\right)$, linked to the surface, in order to use the same orientation for all sinks. This global frame is defined such as $\vec{X} \cdot \overrightarrow{e_{r}}=\cos \phi$. We can then add the contribution of every sink in order to obtain the force received by the object from the flow induced by all the sinks:

$$
\vec{F}_{a i r /\left(O_{s}, \vec{X}, \vec{Y}\right)}=\left[\begin{array}{c}
F_{a i r, x} \\
F_{a i r, y}
\end{array}\right]
$$

where the forces $F_{a i r, x}$ and $F_{a i r, y}$ are given by:

$$
\left\{\begin{array}{l}
F_{\text {air }, x}=\sum_{i=1}^{N}-\frac{b}{2 \pi}\left[f_{1} \cos (\phi+\theta)+f_{2} \sin (\phi-\theta)\right] \Lambda_{i} \\
F_{\text {air }, y}=\sum_{i=1}^{N}-\frac{b}{2 \pi}\left[f_{1} \sin (\phi+\theta)-f_{2} \cos (\phi-\theta)\right] \Lambda_{i}
\end{array}\right.
$$

The angles $\phi$ and $\theta$ and the functions $f_{1}$ and $f_{2}$ are defined for each sink.

2) Moment of forces: As for the force, the moment can be separated in two parts: a first one $\Gamma_{a i r}$ due to the air flow and a second one $\Gamma_{P}$ linked to the velocity of the point $P$ :

$$
\begin{aligned}
\mathcal{M}_{O_{o}} & =\iint \overrightarrow{O_{o} P} \wedge b \overrightarrow{V_{r e l, P}} d S \\
& =\iint-r_{i} \overrightarrow{e_{r}} \wedge b \overrightarrow{V_{\text {air }}}-\iint \overrightarrow{O_{o} P} \wedge b \overrightarrow{V_{P}} d S \\
& =\sum_{i=1}^{N} \frac{b r_{i}}{2 \pi}\left[f_{1} \sin \theta-f_{2} \cos \theta\right] \Lambda_{i}-\frac{b\left(l^{3} L+l L^{3}\right)}{12} \dot{\alpha} \\
& =\Gamma_{\text {air }}-\Gamma_{P}
\end{aligned}
$$

\section{Object's dynamics}

Neglecting the dynamics of the establishment of the flow, the dynamics of the object in the flow is simply:

$$
\left\{\begin{array}{l}
m \ddot{x}=F_{a i r, x}(\Lambda)-F_{P, x}=F_{a i r, x}(\Lambda)-b L l \dot{x} \\
m \ddot{y}=F_{a i r, y}(\Lambda)-F_{P, y}=F_{a i r, y}(\Lambda)-b L l \dot{y} \\
I \ddot{\alpha}=\Gamma_{a i r}(\Lambda)-\Gamma_{P}=\Gamma_{a i r}(\Lambda)-\frac{b\left(l^{3} L+l L^{3}\right)}{12} \dot{\alpha}
\end{array}\right.
$$

where $m$ is the mass of the object and $I$ its moment of inertia.

Our idea is to form a convenient flow according to a desired motion of the object by choosing the appropriate combination of sinks. Then, if we could directly control the values of $F_{a i r, x}, F_{a i r, y}$ and $\Gamma_{a i r}$, the system would be reduced to a first order with integrator.

\section{INVERSE MODELING CONTROL}

The effects of potential air flow are non-linear and coupled but these attributes can be linearized by an inverse model. This section provide a detailed description of the control scheme proposed to perform 3-DOF positioning.

\section{A. Architecture}

In general, the key assumption in direct inverse modeling control is that a plant can be made to track an input command signal when this signal is applied to a controller whose transfer function approximates the inverse of the plant's transfer function.

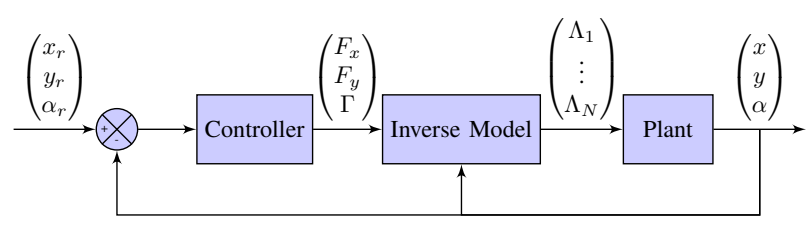

Fig. 2. The inverse modeling control architecture.

The inverse modeling architecture we use here has a feedback loop as depicted in Figure 2 in order to improve its robustness. The controller calculates the forces and torque to apply to the object according to the position errors. Then the inverse filter takes the desired forces and torque as input variables and determines the strength $\Lambda_{i}$ of each suction point as an output variable. If the inverse model is accurate, the composite system (inverse filter + plant) is simply reduced to three independent SISO systems each one being a first order with integrator. This allows simple control designs including PI/PID and LQR designs to be utilized.

The problem that arises with this method is then the inversion of the non-linear model presented in the previous section.

\section{B. Problem statement}

In order to inverse the non-linear model of Eq. (18) and Eq. (19), we have represented it in the matrix form:

$$
\left[\begin{array}{c}
F_{x} \\
F_{y} \\
\Gamma
\end{array}\right]=\left[\begin{array}{cccc}
A_{1,1} & A_{1,2} & \cdots & A_{1, N} \\
A_{2,1} & A_{2,2} & \cdots & A_{2, N} \\
A_{3,1} & A_{3,2} & \cdots & A_{3, N}
\end{array}\right] \times\left[\begin{array}{c}
\Lambda_{1} \\
\vdots \\
\Lambda_{N}
\end{array}\right]
$$

where the components of the $A$ matrix are given by:

$$
\left\{\begin{array}{l}
A_{1, i}=-\frac{b}{2 \pi}\left[f_{1, i} \cos \left(\phi_{i}+\theta_{i}\right)+f_{2, i} \sin \left(\phi_{i}-\theta_{i}\right)\right] \\
A_{2, i}=-\frac{b}{2 \pi}\left[f_{1, i} \sin \left(\phi_{i}+\theta_{i}\right)-f_{2, i} \cos \left(\phi_{i}-\theta_{i}\right)\right] \\
A_{3, i}=\frac{b r_{i}}{2 \pi}\left[f_{1, i} \sin \theta_{i}-f_{2, i} \cos \theta_{i}\right]
\end{array}\right.
$$

Each coefficient $A_{j, i}$ depends on the object position and orientation. So, the $A$ matrix is changing at each sampling period. The inversion process must be done each time. The first requirement for the inversion method is then to be fast enough to run in real time.

Knowing the desired forces and torque and the $A$ matrix, the problem is then to find a solution to a system of linear equations. The first idea is to compute the least squares solution using the singular value decomposition. Unfortunately, the solution is not applicable for two reasons. First, the obtained $\Lambda_{i}$ values will take their values in $\Re$ while the sinks have a maximal suction flux. Secondly, the obtained $\Lambda_{i}$ will be signed that would requires both suction points and admission points that is technically more complex.

As the number of equations is smaller than the number of variables, it may exist a lot of solutions. The first requirement is that solutions must be physically feasible. So, we impose that the $\Lambda_{i}$ values must take their value in $\left[0 ; \Lambda_{\max }\right]$ where $\Lambda_{\max }$ is the maximal volume flux of sinks.

Another requirement is guided by the energy saving: among the solutions, we would like to select the one that consumes the less of air. 


\section{Linear programming}

Considering one control step, finding the $\Lambda_{i}$ values corresponds to solve a linear program. A linear program consists in minimizing or maximizing an objective function defined as a linear combination of problem variables, under a set of linear constraints that have to be satisfied. A well known method for solving such a program was described by Dantzig [15] as the simplex method.

The simplex method allows solving program that are expressed as follows (in the normal form):

$$
\begin{aligned}
\text { minimize } & C^{T} \cdot X \\
\text { such that } & A \cdot X=B \\
\text { where } & X \in \Re^{n} \text { and } X \geq 0 \\
& C \in \Re^{n}, B \in \Re^{m} \text { and } A \in \Re^{m} \times \Re^{n}
\end{aligned}
$$

In our context, finding a control mode at each iteration of the control process can be assimilated to the resolution of the following linear program:

$$
\begin{array}{cc}
\text { minimize } & \sum_{i=1}^{N} \Lambda_{i}, \\
\text { such that } & A \cdot\left[\begin{array}{c}
\Lambda_{1} \\
\vdots \\
\Lambda_{N}
\end{array}\right]=\left[\begin{array}{c}
F_{x} \\
F_{y} \\
\Gamma
\end{array}\right] \\
\text { where } & 0 \leq \Lambda \leq \Lambda_{\max }
\end{array}
$$

As we already said in previous section, one objective is to find a solution that save energy. Then, one way to reach this goal is to minimize $\Lambda_{i}$ values, this can be formulated by the sum of $\Lambda_{i}$. This linear program can be rewritten in the normal form to be solved using the simplex method [15]. Matlab optimization toolbox proposes the linprog function to achieve such a problem.

\section{SOlutions ANALYSiS}

In this section, we analyze some cases of model inversions. The location of the sinks are given by the geometry of the induced air flow surface used for the experimental validation (cf. Section V).

\section{A. Cases study}

Figure 3 depicts the potential air flows obtained using the previously described linear programming for different values of forces and torque. In the first one, the desired value of forces and torque are $F_{x}=0 \mathrm{mN}, F_{y}=0.1 \mathrm{mN}$ and $\Gamma=0 \mathrm{mN}$.m. The objective is to push the object to the north. The result of the linear programming is to open two sinks with medium suction flux and located at the north of the object. The streamlines clearly show that the object will be moved to the north. The second case shows the air flow able to move the object to the east when the object is rotated. The combination of three sinks allow to compensate the orientation of the object. In the third case, the object is pushed five times stronger in the $X$ direction than in the $Y$ direction. The last case illustrates the application of a torque

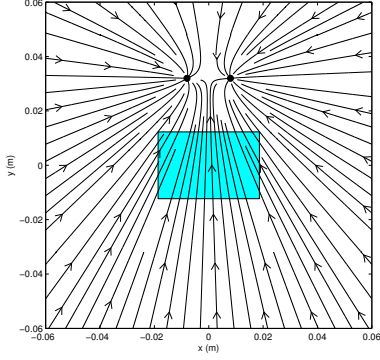

(a) $F_{y}=0.1 \mathrm{mN}$

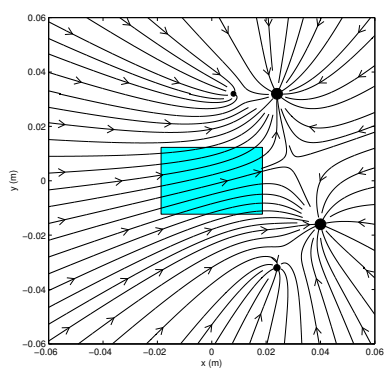

(c) $F_{x}=0.5 \mathrm{mN}, F_{y}=0.1 \mathrm{mN}$

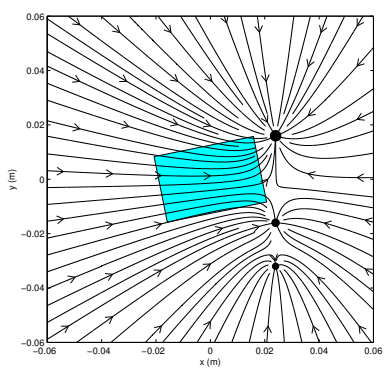

(b) $F_{x}=0.5 \mathrm{mN}$

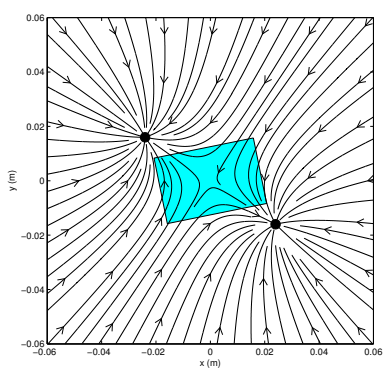

(d) $\Gamma=0.01 \mathrm{mN} . \mathrm{m}$
Fig. 3. Resulting potential air flows and sinks of four cases of model inversion.

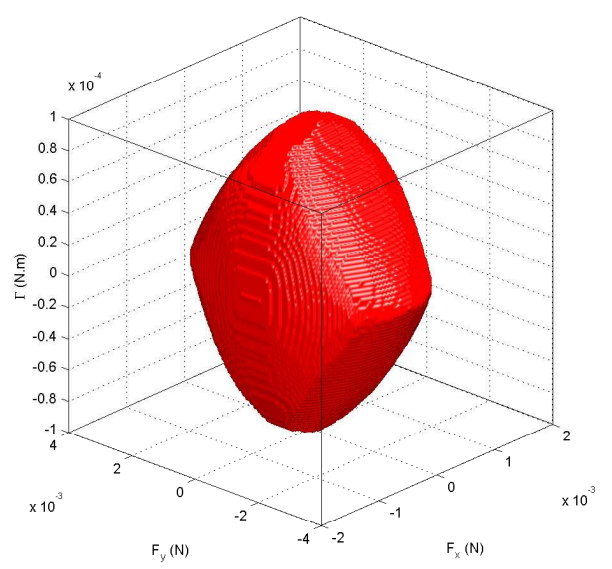

Fig. 4. 3D view of the invertible space for the object located at $x=0 \mathrm{~mm}$, $y=0 \mathrm{~mm}$ and $\alpha=0 \mathrm{rad}$.

to the object. The streamlines clearly show the squeezing effect on the object surface.

For all case, we can see that the solution is very sparing in terms of number of sinks to activate. This is a major interest of linear programming: the provided solution always minimizes the air consumption.

\section{B. Invertible space}

Another advantage of linear programming is that we can know if there is a solution or not. This feature can be used to determine the space in which the inversion is feasible. This "invertible space" is represented in Fig. 4 for the object located at $x=0 \mathrm{~mm}, y=0 \mathrm{~mm}$ and $\alpha=0 \mathrm{rad}$. It is noteworthy that this volume is convex (no holes inside). It means that if we take a point defined by its coordinates $\left[\begin{array}{lll}F_{x} & F_{y} & \Gamma\end{array}\right]^{T}$ inside the boundary of the invertible space, there 


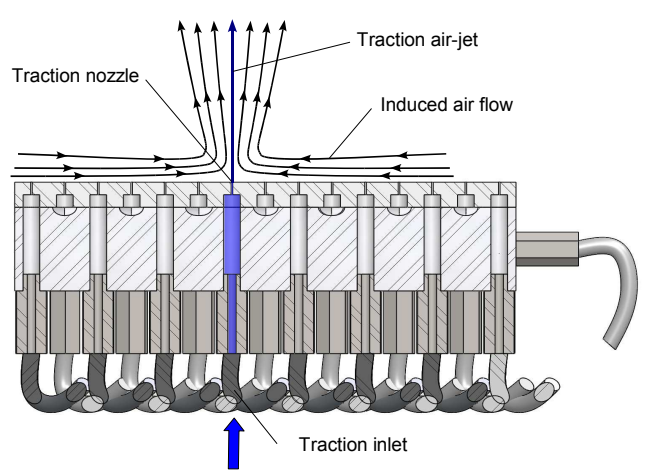

Fig. 5. Cross view of the induced air flow surface showing the generation of the induced air flow (the path of the air flow is darkened).

is a solution to the inversion problem. So the invertible space defines the space within the output values of the controllers must be. This property help us to determine the coefficients of the controllers described in the following.

Moreover it means that we are able to apply the desired forces and torque to the object with any coupling constraints. This property allows us to design three independent controllers, one for the $x$ position, one for the $y$ position and one for the orientation.

\section{EXPERimental Results}

In this section, the control method is validated experimentally thanks to the prototype of an induced air flow surface we have developed in previous works [10], [11], [12].

\section{A. Experimental setup}

The induced air flow surface is a $120 \mathrm{~mm} \times 120 \mathrm{~mm}$ square surface drilled by two kinds of holes. The object is maintained in constant levitation thanks to the air cushion created by the airflow that comes through a common air inlet. The specificity is that the object can be moved on the table by generating strong vertical air jets through specific holes of the surface. These vertical air jets create a suction effect that pulls the object towards the nozzle (cf. Fig. 5). Each nozzle is driven by an independent solenoid valve. Default settings for operating pressures are $10 \mathrm{kPa}$ for levitation and $500 \mathrm{kPa}$ for traction.

The experimental setup for the induced air flow surface is composed of pressurized air supply, two pressure regulators, the set of 56 solenoid valves and its control system, and a computer for vision processing. Fig. 6 describes the complete hardware configuration.

In [12], we identified and validated experimentally the parameters of the model. The object we use here is a rectangular aluminum object which dimensions are $37.3 \mathrm{~mm} \times 24.6 \mathrm{~mm} \times 4.82 \mathrm{~mm}$ and which mass is $12.53 \mathrm{~g}$. The skin friction coefficient of this object is $b=0.8$. The strength of the sinks is $\Lambda_{i}=5.592 e-2 \mathrm{~m}^{2} . \mathrm{s}^{-1}$.

\section{B. 3-DOF position control}

The control architecture detailed in section III has been simulated and tested experimentally. The control signal is

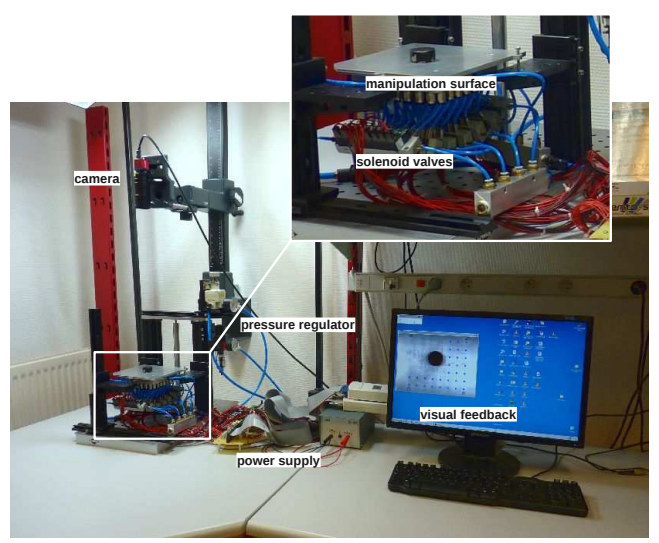

Fig. 6. Overview of the experimental setup.
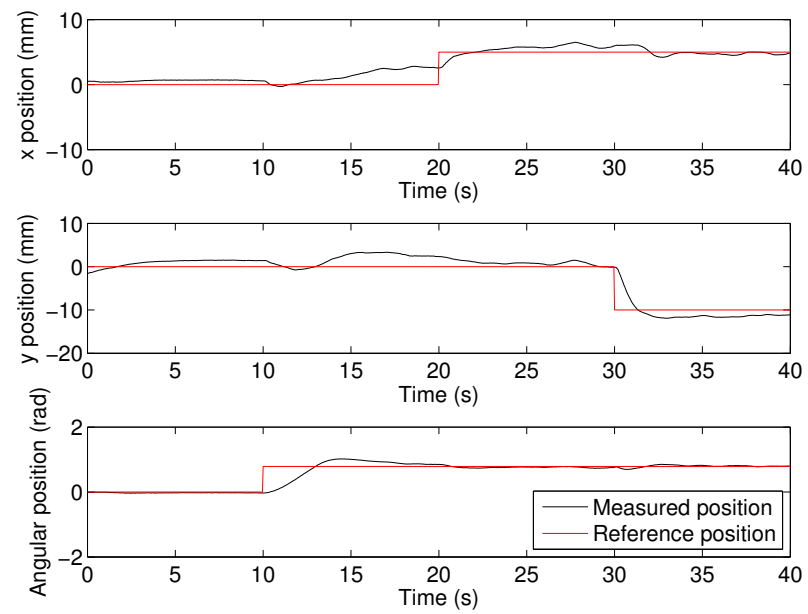

Fig. 7. 3-DOF position tracking.

calculated in real-time at a rate of $30 \mathrm{~Hz}$. At each sampling time, three independent PID controllers evaluate the forces and torque to apply to the object according to the position errors. Then, the $A$ matrix is computed and inverted to activate the correct sinks.

The proportional, integral and derivative coefficients of the three PID controllers have been tuned to obtain the best performances while producing commands inside the invertible space (cf. section IV-B). For the $x$ and $y$ positions, they are respectively: $K_{P}=0.2, K_{I}=0.02$ and $K_{D}=0.2$. For $\alpha$ orientation, they are: $K_{P}=1 \mathrm{e}-4, K_{I}=2 \mathrm{e}-5$ and $K_{D}=1 \mathrm{e}-4$.

Fig. 7 shows experiment results of 3-DOF position tracking. The motion of the object can be further appreciated in the video clip accompanying this paper. ${ }^{1}$. The three degrees of freedom are controlled at the same time that validates the control method developed before. Furthermore, the 3-DOF position tracking is feasible with sinks of same properties: all of them are suction points with a same strength $\Lambda_{\max }$.

In these very first experimental results, the performances of the PID control strongly depend on the external perturbations and on the modeling uncertainties and errors.

\footnotetext{
${ }^{1}$ Also available at http://www.femto-st.fr/ guillaume.laurent/
} 

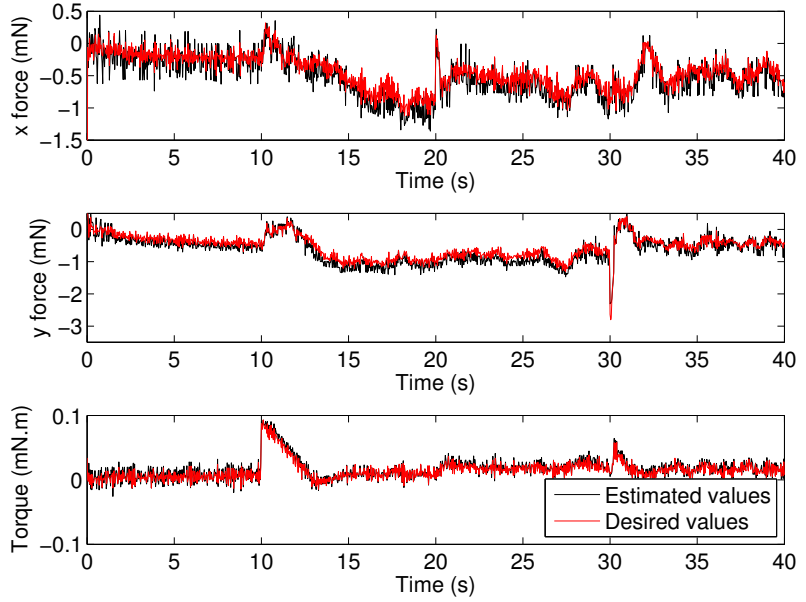

Fig. 8. Desired forces $\left(F_{x}\right.$ and $\left.F_{y}\right)$ and torque $(\Gamma)$ values calculated by the controller and estimated values produced by the sinks activated by the linear programming algorithm.

These results confirms that the three dimensions are nearly uncoupled. At time $10 \mathrm{~s}$, as the reference angular position changes, the object rotates around its center of gravity. We can note a little deviation of $x$ and $y$ positions. At time $20 \mathrm{~s}$, the $x$ reference position changes and the object moves to this position. There is only little effect on the two other positions, as when the $y$ reference position changes at time $30 \mathrm{~s}$. This can be due to the PID controllers tuning that favors for the orientation control performances and to the discretization of the command signal.

\section{Analysis of the discretization effect}

The linear program detailed in section III-C gives solutions with $\Lambda_{i}$ value in $\left[0 ; \Lambda_{\max }\right]$. But experimentally, all the sinks have the same strength as the solenoid valves are on-off ones. These $\Lambda$ values have then to be convert into binary values in $\left\{0, \Lambda_{\max }\right\}$, which correspond respectively to the inactivation and the activation of the air jet. This discretization has been done following this rule: when $\Lambda<\Lambda_{\max } / 10$, the sink is not active (valve closed), and when $\Lambda>\Lambda_{\max } / 10$, the sink is active (valve opened). The threshold of $1 / 10$ has been determined experimentally in order to have the generated values of the forces and torque as close as possible to the desired values. Fig. 8 shows the desired values of these forces and torque and the estimated values (calculated thanks to the model of the surface with the activated sinks) during the previous experiment. The values are very closed so the discretization has only little effect on experimental results. However, the effect could be reduced changing the on-off valves into proportional ones and controlling precisely the strength $\Lambda_{i}$ of each sink.

\section{CONCLUSIONS AND FutURE WORKS}

In this paper, we have proposed a general method able to perform 3-DOF position control of objects with potential air flow manipulators. This method is based on the inversion of the model of the velocity flow field depending on the spatial configuration of vertical sinks. The controller defines the forces and torque to apply to the object in order to move it to the desired position. Then, a linear programming algorithm determines which sinks to activate in order to produce these desired forces and torque. This algorithm uses the simplex method which finds the solution that minimizes the air consumption. This general method of 3-DOF position control have been validated experimentally on an induced air flow surface we have developed before. First experiments have been done with basic PID controllers in order to validate the inverse modeling control method: the three degrees of freedom of an object has been controlled at the same time. As the performances of this method strongly depends on the modeling uncertainties and on the perturbations, the robustness could be improved in future works designing an internal model controller. We could also obtain better control performances reducing the discretization effects on the forces and torque than can be generated. For example, a solution is to change the on-off solenoid valves into proportional valves in order to control more precisely the strength of the suction points.

\section{REFERENCES}

[1] M. Hoetzle, T. Dunifon, and L. Rozevink. Glass transportation system. U.S. Patent 6,505,483, 2003.

[2] Andrew Berlin, David Biegelsen, Patrick Cheung, Markus Fromherz, David Goldberg, Warren Jackson, Bryan Preas, James Reich, and LarsErik Swartz. Motion control of planar objects using large-area arrays of mems-like distributed manipulators. In Micromechatronics, 2000.

[3] J. van Rij, J. Wesselingh, R. A. J. van Ostayen, J.W. Spronck, R.H. Munnig Schmidt, and J. van Eijk. Planar wafer transport and positioning on an air film using a viscous traction principle. Tribology International, 42:1542-1549, 2009.

[4] Y. Fukuta, Y.-A. Chapuis, Y. Mita, and H. Fujita. Design, fabrication and control of mems-based actuator arrays for air-flow distributed micromanipulation. IEEE/ASME Journal of Microelectromechanical Systems, 15(4):912-926, 2006.

[5] Rabah Zeggari, Reda Yahiaoui, Julien Malapert, and Jean-François Manceau. Design and fabrication of a new two-dimensional pneumatic micro-conveyor. Sensors \& Actuators: A.Physical, 164:125-130, 2010.

[6] Jonathan Luntz and Hyungpil Moon. Distributed manipulation with passive air flow. In Proc. of the IEEE Int. Conf. on Intelligent Robots and Systems, pages 195-201, 2001.

[7] Hyungpil Moon and Jonathan Luntz. Distributed manipulation of flat objects with two airflow sinks. IEEE Transactions on robotics, 22(6):1189-1201, 2006.

[8] Konstantinos Varsos and Jonathan Luntz. Superposition methods for distributed manipulation using quadratic potential force fields. IEEE Transactions on robotics, 22(6):1202-1215, 2006.

[9] Konstantinos Varsos, Hyungpil Moon, and Jonathan Luntz. Generation of quadratic potential force fields from flow fields for distributed manipulation. IEEE Transactions on robotics, 22(1):108-118, 2006.

[10] Anne Delettre, Guillaume J. Laurent, and Nadine Le Fort-Piat. A new contactless conveyor system for handling clean and delicate products using induced air flows. In Proc. of the IEEE Int. Conf. on Intelligent Robots and Systems, pages 2351-2356, 2010.

[11] Guillaume J. Laurent, Anne Delettre, and Nadine Le Fort-Piat. A new aerodynamic traction principle for handling products on an air cushion. IEEE Transactions on robotics, 27:2, 2011.

[12] Anne Delettre, Guillaume J. Laurent, and Nadine Le Fort-Piat. 2-dof contactless distributed manipulation using superposition of induced air flows. In Proc. of the IEEE Int. Conf. on Intelligent Robots and Systems, pages 5121-5126, 2011.

[13] G. N. Abramovich. The theory of turbulent jets. MIT Press, 1963.

[14] Frank M. White. Fluid Mechanics. McGraw-Hill Science/Engineering/Math, 2002.

[15] George Bernard Dantzig. Linear Programming and Extensions. RAND Corporation, 1963 